Non-uniformly polarized beams across their transverse profiles: an introductory study for undergraduate optics courses

This article has been downloaded from IOPscience. Please scroll down to see the full text article.

2004 Eur. J. Phys. 25793

(http://iopscience.iop.org/0143-0807/25/6/011)

View the table of contents for this issue, or go to the journal homepage for more

Download details:

IP Address: 147.96.14.16

The article was downloaded on 11/06/2013 at 17:55

Please note that terms and conditions apply. 


\title{
Non-uniformly polarized beams across their transverse profiles: an introductory study for undergraduate optics courses
}

\author{
Gemma Piquero and Javier Vargas-Balbuena
}

Departamento de Óptica, Facultad de Ciencias Físicas, Universidad Complutense de Madrid, 28040 Madrid, Spain

Received 3 March 2004, in final form 7 July 2004

Published 14 September 2004

Online at stacks.iop.org/EJP/25/793

doi:10.1088/0143-0807/25/6/011

\begin{abstract}
We provide a simple theoretical study of beams non-uniformly polarized across their transverse sections which can be introduced in undergraduate optics courses. In order to generate such beams we propose to use a slightly convergent (or divergent) linearly and uniformly polarized beam impinging on an anisotropic uniaxial material with the beam propagation direction along the optic axis. Analytical expressions for the Jones vector, Stokes parameters, ellipticity and azimuth at each point of the transverse section, perpendicular to the propagation direction, are obtained at the output of this system. By means of these parameters a detailed description of the state of polarization across the transverse profile is given.
\end{abstract}

(Some figures in this article are in colour only in the electronic version)

\section{Introduction}

Polarization of light waves is one of the main topics in optics and many papers related to this subject are currently published. In undergraduate studies, the concept of polarization of light is introduced together with different representations, such as Jones vectors for totally polarized light, Stokes parameters and the degree of polarization for partially polarized light [1,2]. Some didactical papers about these representations and parameters have appeared in the literature (see, for example, [3-7]). Generally, in these works it is assumed that fields are uniformly polarized across their transverse sections, perpendicular to the propagation direction. This means that all points of the transverse profile have the same state and degree of polarization.

In recent years, some investigations have shown that several kinds of laser exist with a non-uniform distribution of polarization across their transverse section: examples are radial 
or azimuthal distributions [8-12]. On the other hand, there are a lot of optical systems which transform uniformly polarized beams into non-uniform ones, for example by propagation through a birefringent Nd:YAG rod [13], calcite crystal [14] or through polarization gratings $[15,16]$, etc. These beams present special and interesting properties. For example, the polarization distribution does not remain constant upon free propagation and it can be changed by means of several optical systems.

One of the simplest methods to obtain a non-uniform distribution of the polarization state across the transverse section is to use an anisotropic crystal such as KDP, quartz, calcite, etc. When a totally, linearly and uniformly polarized beam, slightly divergent (or convergent), impinges on an anisotropic material with the mean propagation direction along its optic axis, we obtain a non-uniform distribution of the polarization in the transverse profile of the output beam. This same set-up is used in conoscopy [17], which is one of the interferometric methods for the study of birefringent crystals in optics, crystallography and mineralogy, which are undergraduate courses in physics and geology. On the other hand, birefringence by a uniaxial crystal is a basic topic in undergraduate courses of physics or engineering studies and several optical devices are based on linear and non-linear effects in birefringent crystals (Pockels, Kerr, etc). When these subjects are studied it presents a good chance to introduce a first study of beams whose polarization state varies from one point to another across the transverse section.

The purpose of this work is to present an introductory study of non-uniformly polarized beams generated upon propagation through anisotropic materials in which the optic axis coincides with the beam propagation direction (in our case, the z-axis). We analytically obtain the Jones vector, the intensity, the Stokes parameters, the azimuth and ellipticity of the light at the output of the anisotropic material. For simplicity, we will use the paraxial approximation. Within this approximation we can neglect the longitudinal component of the beam and consequently we will work only with the transverse components of the field $E_{x}$ and $E_{y}$. Additionally, we will omit the reflection and transmission factors at the two faces of the medium. On the other hand, depolarizing effects will not be taken into account, so we can use the Jones formalism. The Jones vector at the output of the system is obtained by calculating the optical paths of the $x$ and $y$ components of the field. From this vector the rest of the parameters may be deduced. In the following section we derive, step by step, the Jones vector at the output of our system. By using a polarizer whose transmission axis is perpendicular to the polarization of the incident beam we reproduce the well-known 'cross-shaped intensity distribution' or also called 'interference figure with polarized light'. In section 3, the Stokes parameters of the output beam and related parameters such as the azimuth and ellipticity are obtained, and an analysis and discussion of the results is given.

\section{Jones vector}

Let us begin with a quasi-monochromatic, totally uniformly polarized plane wave propagating along the $z$-axis, whose state of polarization is linear along the $x$-direction, represented by the Jones vector [2],

$$
\mathbf{E}^{i}(x, y, z)=E_{0}(x, y)\left(\begin{array}{l}
1 \\
0
\end{array}\right) \exp (\mathrm{i} w t) \exp (-\mathrm{i} k z)
$$

where the superscript ' $i$ ' indicates incident beam, $w$ is the frequency and $E_{0}$ is the amplitude of the field. We will assume a more realistic case in which the amplitude is not constant but a 


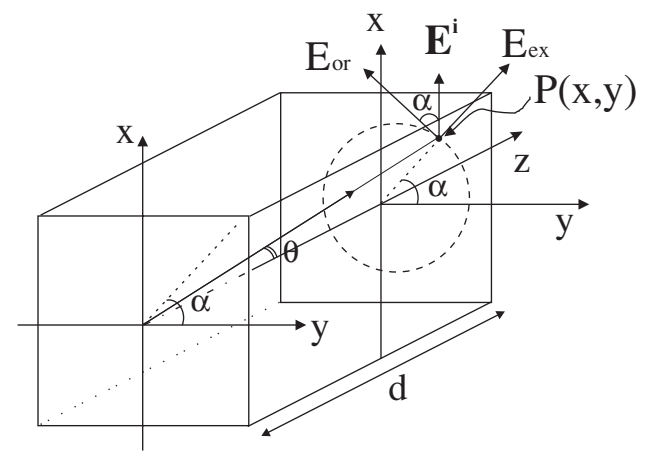

Figure 1. Schematic diagram of the reference system used. $\mathbf{E}^{\mathrm{i}}$-electric field of the incident beam, $E_{\text {or }}$-ordinary component, $E_{\mathrm{ex}}$ - extraordinary component, $d$-length of the anisotropic medium, $\alpha$-angle that forms the perpendicular of the principal section with the $x$-axis and $\theta$ is the angle of propagation of a ray with respect to the $z$-axis

Gaussian function as

$$
E_{0}(x, y)=\frac{1}{\sqrt{\sigma}(2 \pi)^{1 / 4}} \exp \left(-\frac{x^{2}+y^{2}}{4 \sigma^{2}}\right)
$$

with $\sigma$ being proportional to the beam width. As the temporal factor does not change upon propagation through the anisotropic material, we will omit it in the following.

The anisotropic material, with length $d$ and optic axis oriented along the propagation direction, is characterized by two refractive indices, the ordinary index, $n_{o}$, and the extraordinary one, $n_{e}$. If the incident beam is collimated parallel to the optic axis, the material behaves as an isotropic crystal and the polarization state does not change at the output of the medium. For any other direction, which forms an arbitrary angle $\theta$ with the optic axis of the crystal (see figure 1), the beam may be decomposed inside the material into two components, the ordinary, $E_{\mathrm{or}}$, and the extraordinary one, $E_{\mathrm{ex}}[1]$. Just at the input face $(z=0)$ of the anisotropic medium we have

$$
\mathbf{E}^{\prime i}(x, y)=\left(\begin{array}{c}
E_{\text {or }} \\
E_{\mathrm{ex}}
\end{array}\right)=E_{0}(x, y)\left(\begin{array}{c}
\cos \alpha \\
\sin \alpha
\end{array}\right),
$$

where $\alpha$ is the angle between the incident field, $\mathbf{E}^{i}$, at the point $P$ and the direction perpendicular to the principal section (defined as the plane that contains the optic axis $(z)$ ) and the propagation direction inside the material (see figure 1)).

At the output of the anisotropic material (indicated by the superscript ' $o$ ') we have that each component has changed as [1]

$$
\begin{aligned}
& E_{\mathrm{or}}^{o}=E_{\mathrm{or}} \exp \left(-\mathrm{i} k n_{o} l_{\mathrm{or}}\right), \\
& E_{\mathrm{ex}}^{o}=E_{\mathrm{ex}} \exp \left(-\mathrm{i} k n_{e}^{\prime} l_{\mathrm{ex}}\right),
\end{aligned}
$$

where $l_{\text {or }}$ and $l_{\text {ex }}$ are the travelled distances by each component in the anisotropic medium. Note that in the above expression we have written $n_{e}^{\prime}$ with the prime to indicate that this refractive index depends on the angle $\theta$. This refractive index is related to the indices $n_{e}$ and $n_{o}$ by the expression

$$
1 / n_{e}^{\prime 2}=\left(1 / n_{o}^{2}\right) \cos ^{2} \theta+\left(1 / n_{e}^{2}\right) \sin ^{2} \theta
$$

Up to now we have considered birefringence, which means each component travels different lengths inside the material. However, if the angle $\theta$ is small (paraxial approximation) we can 
set $l_{\text {or }} \approx l_{\text {ex }} \approx l$, where $l$ is the distance travelled by the beam. Then the output electric field may be written as

$$
\mathbf{E}^{o}(x, y)=\left(\begin{array}{l}
E_{\mathrm{or}} \exp \left(-\mathrm{i} k n_{o} l\right) \\
E_{\mathrm{ex}} \exp \left(-\mathrm{i} k n_{e}^{\prime} l\right)
\end{array}\right) .
$$

In the $x y$ reference system (see figure 1) the Jones vector of the output beam becomes

$$
\mathbf{E}^{o}(x, y)=\left(\begin{array}{c}
E_{\mathrm{or}} \exp \left(-\mathrm{i} k n_{o} l\right) \cos \alpha+E_{\mathrm{ex}} \exp \left(-\mathrm{i} k n_{e}^{\prime} l\right) \sin \alpha \\
E_{\mathrm{ex}} \exp \left(-\mathrm{i} k n_{e}^{\prime} l\right) \cos \alpha-E_{\mathrm{or}} \exp \left(-\mathrm{i} k n_{o} l\right) \sin \alpha
\end{array}\right),
$$

and, using equation (3) and the relations

$$
\begin{aligned}
& x=r \cos \alpha, \quad y=r \sin \alpha, \\
& \mathbf{E}^{o}(x, y)=E_{0}(x, y)\left(\begin{array}{l}
\frac{x^{2}}{r^{2}} \exp \left(-\mathrm{i} k n_{o} l\right)+\frac{y^{2}}{r^{2}} \exp \left(-\mathrm{i} k n_{e}^{\prime} l\right) \\
\frac{x y}{r^{2}}\left[\exp \left(-\mathrm{i} k n_{e}^{\prime} l\right)-\exp \left(-\mathrm{i} k n_{o} l\right)\right]
\end{array}\right) .
\end{aligned}
$$

As can be seen from equation (10) we have obtained a totally polarized beam whose state of polarization changes from one point to another across the transverse profile. To see that, let us remember that the Jones vector of a uniformly polarized beam is usually written as

$$
\mathbf{E}=\left(\begin{array}{c}
E_{x} \\
E_{y}
\end{array}\right) \propto\left(\begin{array}{c}
A \exp \left(-\mathrm{i} \delta_{x}\right) \\
B \exp \left(-\mathrm{i} \delta_{y}\right)
\end{array}\right)
$$

with $A, B, \delta_{x}$ and $\delta_{y}$ constants. Depending on the values of these parameters we have linear, circular or elliptically polarized beams. In contrast, equation (10) $E_{x}$ and $E_{y}$ depend on the coordinates $x$ and $y$. Consequently, the Jones vector changes from one point to another, leading to different state of polarization at each point of the transverse section.

When at the output of the anisotropic medium we place a polarizer with the transmission axis along the $y$-axis, the analytical expression of the intensity at the output of the medium is given by

$$
I(x, y) \propto\left|\mathbf{E}_{o}(x, y)\right|^{2}=E_{0}^{2} \frac{4 x^{2} y^{2}}{\left(x^{2}+y^{2}\right)^{2}} \sin ^{2}(\delta / 2),
$$

which has been derived by setting the $x$-component equal to zero in equation (10) and where $\delta=\delta_{y}-\delta_{x}$.

The phase difference between both components is due to the different refractive indices, $\delta=k\left(n_{e}^{\prime}-n_{o}\right) l$. Using equation (6) we get

$$
1 / n_{e}^{\prime 2}-1 / n_{o}^{2}=\left(1 / n_{e}^{2}-1 / n_{o}^{2}\right) \sin ^{2} \theta .
$$

When the difference between the refractive indices in comparison with their values is small we can make the approximation [1]

$$
n_{e}^{\prime}-n_{o} \approx\left(n_{e}-n_{o}\right) \sin ^{2} \theta .
$$

The value of $\sin ^{2} \theta$ can be written as a function of the coordinates $(x, y, z=d)$ (figure 1) and $\delta$ reads

$$
\delta=k\left(n_{e}-n_{o}\right) \frac{x^{2}+y^{2}}{\sqrt{x^{2}+y^{2}+d^{2}}} .
$$

Equation (12) has been plotted in figure 2 as a contour map. We obtain the typical figure called an 'interferential diagram with polarized light' [1]. The fact that the intensity after the polarizer is not constant across the transverse section of the output beam indicates that the polarization state changes from one point to another. Nevertheless, the beam itself remains totally polarized. 

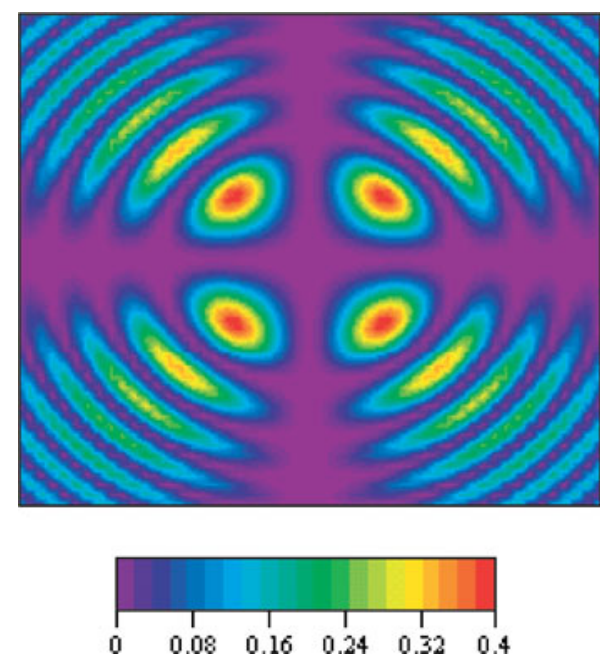

Figure 2. Intensity at the output of a polarizer whose transmission axis is perpendicular to the incident beam. Length of the anisotropic medium $d=20 \mathrm{~mm}, \lambda=633 \mathrm{~nm}, \sigma=1 \mathrm{~mm}$, material $-\mathrm{KH}_{2 \mathrm{PO} 4}(\mathrm{KDP}), n_{o}=1.5079$ and $n_{e}=1.4673$. Size of the picture $1 \mathrm{~mm} \times 1 \mathrm{~mm}$.

\section{Stokes parameters}

In order to have a deeper knowledge of the polarization characteristics of the output beam it is useful to calculate the Stokes parameters and the degree of polarization $P$ using the following relationships for quasi-monochromatic fields [1],

$$
\begin{aligned}
& s_{0}=\left\langle E_{x} E_{x}^{*}\right\rangle+\left\langle E_{y} E_{y}^{*}\right\rangle, \\
& s_{1}=\left\langle E_{x} E_{x}^{*}\right\rangle-\left\langle E_{y} E_{y}^{*}\right\rangle, \\
& s_{2}=\left\langle E_{x} E_{y}^{*}\right\rangle+\left\langle E_{x}^{*} E_{y}\right\rangle, \\
& s_{3}=\mathrm{i}\left(\left\langle E_{x} E_{y}^{*}\right\rangle-\left\langle E_{x}^{*} E_{y}\right\rangle\right), \\
& P=\sqrt{\frac{s_{1}^{2}+s_{2}^{2}+s_{3}^{2}}{s_{0}^{2}}},
\end{aligned}
$$

where the bracket means a temporal average (in our case this temporal average is 1). The first Stokes parameter gives us the irradiance of the beam. The second Stokes parameter, $s_{1}$, represents the excess in intensity of light transmitted by a polarizer whose transmission axis is $0^{\circ}$, over the light transmitted by a polarizer with the transmission axis at $90^{\circ}$, both angles measured with respect to the $x$-axis. The parameter $s_{2}$ has a similar representation with respect to the angles $45^{\circ}$ and $135^{\circ}$. Finally $s_{3}$ is equal to the excess in intensity of light with right-handed circular polarization, over that transmitted with left-handed circular polarization.

In our study, the Stokes parameters (equations (16)-(19)) must be evaluated at each point $(x, y)$ of the transverse section of the beam. From equation (10) the following simple analytical equations result:

$$
\begin{aligned}
& s_{0}(x, y)=\left|E_{0}(x, y)\right|^{2}, \\
& s_{1}(x, y)=s_{0}(x, y)\left[\left(\frac{x^{2}}{r^{2}}-\frac{y^{2}}{r^{2}}\right)^{2}+4 \frac{x^{2} y^{2}}{r^{4}} \cos 2 \delta\right],
\end{aligned}
$$


(a)
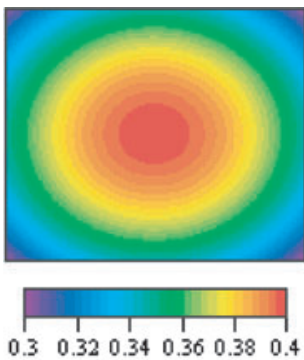

(c)
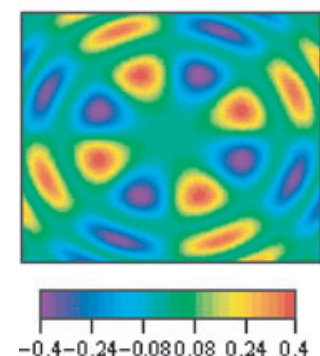

(b)

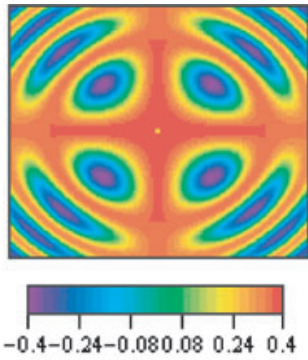

(d)

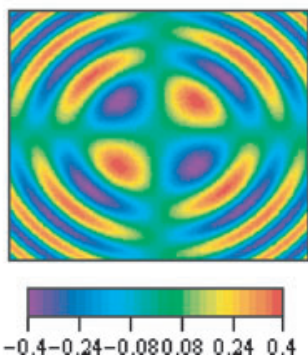

Figure 3. Stokes parameters across the transverse section of the beam. (a) $s_{0}(x, y)$, (b) $s_{1}(x, y),\left(\right.$ c) $s_{2}(x, y)$ and (d) $s_{3}(x, y) . \lambda=633 \mathrm{~nm}, \sigma=1 \mathrm{~mm}$, material- $\mathrm{KH}_{2} \mathrm{PO}_{4}(\mathrm{KDP})$, $n_{o}=1.5079, n_{e}=1.4673$ and $d=20 \mathrm{~mm}$. Size of the pictures $1 \mathrm{~mm} \times 1 \mathrm{~mm}$.

$$
\begin{aligned}
& s_{2}(x, y)=s_{0}(x, y)\left[2\left(\frac{x^{3} y}{r^{4}}-\frac{y^{3} x}{r^{4}}\right)^{2}(1-\cos 2 \delta)\right], \\
& s_{3}(x, y)=s_{0}(x, y)\left[\left(\frac{x y^{3}}{r^{4}}+\frac{x^{3} y}{r^{4}}\right)^{2} 2 \sin 2 \delta\right] .
\end{aligned}
$$

By substituting equations (21)-(24) into equation (20) the degree of polarization turns out to be $P=1$ at each point. $P$ has not changed after propagation through the anisotropic medium, only the local state of polarization has been modified. We have obtained a non-uniformly totally polarized (NUTP) profile.

Stokes parameters have been plotted in figure 3. The parameter $s_{0}(x, y)$ corresponds to the intensity at each point of the transverse section and, in our case, has a Gaussian profile. The other parameters vary also from point to point, showing a non-uniform pattern of polarization state in such a plane. At each point in the transverse section of the output beam, the state of polarization will be, in general, elliptical.

In order to analyse in detail the local state of polarization it is useful to calculate two additional parameters, the ellipticity $\chi$ and the azimuth $\psi$, defined by the expressions [1]

$$
\begin{aligned}
& \chi=\frac{1}{2} \arcsin \left[\frac{s_{3}}{s_{0}}\right], \\
& \psi=\frac{1}{2} \arctan \left[\frac{s_{2}}{s_{1}}\right] .
\end{aligned}
$$

The ellipticity represents the ratio of the minor and major axes satisfying $-\pi / 4<\chi \leqslant \pi / 4$. Positive or negative values of $\chi$ indicate that the polarization is right- or left-handed respectively. The value of $\chi=0$ indicates a linearly polarized beam and $\chi=\pi / 4$ circularly polarized light. 
(a)
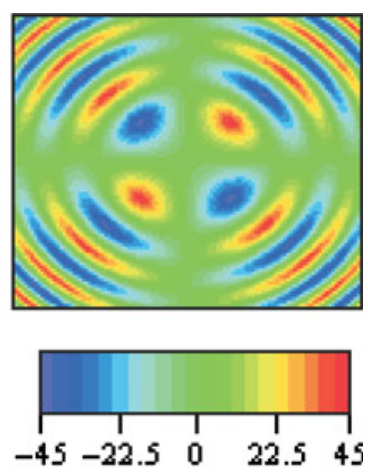

(b)

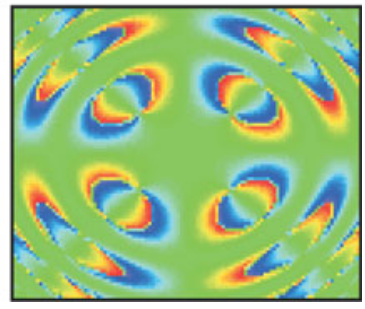

Figure 4. Ellipticity $\chi(x, y)$ and azimuth $\psi(x, y)$ after propagating a distance $d=20 \mathrm{~mm}$ in the KDP medium. $\lambda=633 \mathrm{~nm}, \sigma=1 \mathrm{~mm}$, material- $\mathrm{KH}_{2} \mathrm{PO}_{4}(\mathrm{KDP}), n_{o}=1.5079$ and $n_{e}=1.4673$. Size of the picture $1 \mathrm{~mm} \times 1 \mathrm{~mm}$. The grey/colour scale is the same for both pictures.

The azimuth is the angle between the major axis and the $x$-axis, $\psi$, and satisfies $0 \leqslant \psi<\pi$.

We have evaluated the ellipticity and the azimuth at each point $(x, y)$ of the transverse section of the beam by substituting equations (21)-(24) into equations (25) and (26), resulting in

$$
\begin{aligned}
& \chi(x, y)=\frac{1}{2} \arcsin \left[\frac{2 x y \sin 2 \delta}{x^{2}+y^{2}}\right] \\
& \psi(x, y)=\frac{1}{2} \arctan \left[\frac{4 x y\left(x^{2}-y^{2}\right) \sin ^{2} \delta}{\left(x^{2}-y^{2}\right)^{2}+4 x^{2} y^{2} \cos 2 \delta}\right] .
\end{aligned}
$$

Equations (27) and (28) have been plotted in figure 4. Each value of the ellipticity and the azimuth is represented by a grey level (a colour level in electronic version) in the scale. The beam is linearly polarized when $2 x y \sin 2 \delta=0$, which is satisfied for the axis $x=0$ or $y=0$ or when the phase difference is $\delta=m \pi / 2$ or $\delta=m \pi$ with $m$ an integer. In figure 4(a) these cases correspond to the grey level (or colour) marked in the scale with zero. The polarization state will be circular when the condition $\pm 2 x y \sin (2 \delta)=x^{2}+y^{2}$ is satisfied, for example, when $x= \pm y$ and $\delta=(2 m+1) \pi / 4$. In figure 4(a) these points are on the diagonal and they can be right-handed (value $+45^{\circ}$ ) or left-handed polarized light (value $-45^{\circ}$ ). For the other points we have elliptical polarization.

The other parameter, $\psi$, gives us the azimuth. It should be noted that negative values of this parameter are equivalent to positive ones after adding $\pi$ radians to the angle. The azimuth will be equal to $0^{\circ}$ when $x=0$ or $y=0$. Also it will be $0^{\circ}$ for $x= \pm y$ but with $\delta \neq(2 m+1) \pi / 4$, otherwise the beam would be circularly polarized. For the rest of the 
points the polarization is elliptical with different orientations of the major axis. It will be at $-45^{\circ}=135^{\circ}$ in some regions (see values of the scale) and at $45^{\circ}$ in others.

\section{Conclusions}

Recently, there has been increasing interest in beams with a non-uniform state and degree of polarization across their transverse sections. To introduce the concept of non-uniformly polarized beams, suitable for undergraduate optics courses, a simple study of a particular case is presented. Starting from a basic formalism to describe a beam and the concept of birefringence, usually introduced in undergraduate optics courses, we have derived analytical expressions for the Jones vector, Stokes parameters, azimuth and ellipticity at each point of a transverse section of a beam, initially linearly polarized, after travelling through an anisotropic medium (a KDP crystal). We think that this concept can be introduced to students in an easy way and the study of realistic cases in which polarization is non-uniform provides a good practical argument and exercise for optics courses.

\section{Acknowledgment}

One of the authors (GP) acknowledges support from project BFM2001-1356 of the Ministerio de Ciencia y Tecnología of Spain.

\section{References}

[1] Born M and Wolf E 1999 Principles of Optics 7th edn (Cambridge: Cambridge University Press) chapter 15

[2] Fowles G R 1975 Introduction to Modern Optics (New York: Dover)

[3] Collett E 1968 The description of polarization in classical physics Am. J. Phys. 36 713-26

[4] Hecht E 1970 Note on an operational definition of the Stokes parameters Am. J. Phys. 38 1156-8

[5] Baylis W E, Bonenfant J, Derbyshire J and Huschilt J 1993 Light polarization: a geometric-algebra approach Am. J. Phys. 61 534-45

[6] Auerbach D 2000 Optical polarization without tools Eur. J. Phys. 21 13-7

[7] Dettwiller L 2001 Interpretation and generalization of polarized interferences by means of the Poincaré sphere Eur. J. Phys. 22 576-86

[8] Tidwell S C, Ford D H and Kimura W D 1990 Generating radially polarized beams interferometrically Appl. Opt. $292234-9$

[9] Erdogan T and Hall D G 1990 Circularly symmetric distributed feedback semiconductor laser: an analysis J. Appl. Phys. 68 1435-44

[10] Erdogan T, King O, Wicks G W, Hall D G, Anderson E and Rooks M J 1992 Circularly symmetric operation of a concentric-circle-grating surface emitting AlGaAs/GaAs quantum well semiconductor laser Appl. Phys. Lett. 60 1921-3

[11] Tidwell S C, Kim G H and Kimura W D 1993 Efficient radially polarized laser beam generation with a double interferometer Appl. Opt. 32 5222-29

[12] Freund I 2001 Polarization flowers Opt. Commun. 199 47-63

[13] Martías P M, Mejínez-Herrero R, Piquero G and Movilla J M 2002 Parametric characterization of the spatial structure of non-uniformly polarized laser beams Prog. Quantum Electron. 26 65-130

[14] Provenziani D, Ciattoni A, Cincotti G, Palma C, Ravaccia F and Sapia C 2002 Stokes parameters of a Gaussian beam in a calcite crystal Opt. Express 10 699-706

[15] Gori F 1999 Measuring Stokes parameters by means of a polarization grating Opt. Lett. 24 584-86

[16] Someda C G 2000 Far field of polarization gratings Opt. Lett. 25 1657-9

[17] Pekka H A, Friberg A T, Kailova M A J and Salomaa M M 1999 Conoscopy interferometry of surface-acousticwave substrate crystals Appl. Opt. 38 5399-407 